Check for updates

Cite this: RSC Adv., 2017, 7, 44598

Received 18th July 2017

Accepted 12th September 2017

DOI: 10.1039/c7ra07881a

rsc.li/rsc-advances

\title{
A thermal-fluid coupling numerical study on the characteristics of air-oil two phase flow and heat transfer in a micro UAV bearing chamber
}

\begin{abstract}
Peng Lu, (D) *a Xingwen Zheng, ${ }^{a}$ Wei Li, ${ }^{\text {ac }}$ Peijie Yang, ${ }^{a}$ Hulin Huang ${ }^{* b}$ and Yezhen $\mathrm{Yu}^{\mathrm{d}}$
Design of lubrication systems, particularly of bearing chambers, requires a sufficient understanding of the physical characteristics of the air-oil two phase flow. Due to a high shaft rotating speed of micro UAV (Unmanned Aerial Vehicle) engines, the heat production of bearings often results in a poor cooling effect on the bearings. In the present work, numerical simulation was conducted on the above topic for a certain type of UAV engine. Firstly, the heat production in the bearings was theoretically analysed, and a three dimensional structural model of the bearing chamber was built by Unigraphics (UG) software. Then on the FLUENT platform, some key physical fields, such as path lines, velocity and temperature distributions were presented and discussed, with the simulated results were verified by measured values under typical working conditions. Afterwards, the influences of some important operating conditions, including revolving speed, oil supply, and air inlet velocity were investigated. Finally, optimal values were suggested for each operating condition, taking into consideration the cooling effect, lubrication effect and engine efficiency.
\end{abstract}

\section{Introduction}

The bearing chamber is one of the crucial components in the lubrication system of an aero engine. In this chamber, due to the shaft rotation, the lubricating oil mixes with sealing air flow and they form a complicated air-oil two phase flow. Highpressure and high-temperature working conditions, along with the frictional heat production in bearings, lead to very complex characteristics of flow and heat transfer inside. The lubrication oil plays an important role in lubricating and heat removal, which is beneficial to bearing life and operation; however, the oil may coke or even burn if the temperature is excessively high, which consequently degrades the safety and reliability of the aero engine. Therefore, understanding the characteristics of air-oil two phase flow and heat transfer in the bearing chamber is of great significance for stable and efficient operation of an aero engine.

Adequately resolving the airflow field is important to subsequent computational modeling of two-phase fluids transport and heat transfer characteristics. C. W. Lee et al. ${ }^{1}$

${ }^{a}$ Key Laboratory of Thermal Environment and Thermal Structure of Ministry of Industry and Information Technology, Jiangsu Province Key Laboratory of Aerospace Power System, College of Energy and Power Engineering, Nanjing University of Aeronautics and Astronautics, Nanjing 210016, China. E-mail: plu@nuaa.edu.cn

${ }^{b}$ College of Astronautics, Nanjing University of Aeronautics and Astronautics, Nanjing 210016, China.E-mail: hlhuang@nuaa.edu.cn

'Ming Yang Smart Energy Group Co., Ltd., Zhongshan 528400, China

${ }^{d}$ School of Energy and Mechanical Engineering, Nanjing Normal University, Nanjing 210042, China investigated the single-phase velocity field of a model aero engine bearing chamber, with comparison of computational fluid dynamics (CFD) with particle image velocimetry (PIV) data. Similarly, J. Aidarinis et al. ${ }^{2}$ examined the air-flow field development in an aero engine front bearing chamber, by combination of experimental measurements and CFD modeling.

Besides, the movement of lubrication oil droplet also has strong impacts on the two phase flow and heat transfer inside the bearing chamber. In order to obtain the composition of the liquid (oil) and gas (air) phases, M. Farrall et al. ${ }^{3}$ computed the air/oil flow in a commercial aero engine bearing chamber, with focus on the flow exiting the chamber, which showed good agreement with available experimental data. To describe the effect of oil droplet deformation on the motion, B. Chen et al. ${ }^{4}$ proposed a modified droplet deformation model, by which he calculated the droplet deformation, motion, and the associated mass and momentum transfer in an aero engine bearing chamber. Based on the oil film force balance analysis and wave theory, H. C. Sun et al. ${ }^{5}$ calculated Sauter Mean Diameter (SMD) of the oil droplet, as functions of the rotating disk radius, rotating speed, oil flow rate and oil properties; later on, by adding temperature solution to the oil droplet motion analysis, they further promoted the calculation accuracy of the droplet trajectory, velocity, and temperature. ${ }^{6}$

Furthermore, the oil film, which is very important for the bearing chamber lubrication and heat transfer calculation, is another research topic. M. Farrall et al. ${ }^{7}$ developed a two-phase numerical modeling approach to predict air, oil droplet and oil film behavior in an aero engine bearing chamber including 
strong droplet/film interactions. P. Gorse et al. ${ }^{8}$ focused on the experimental determination of the film thickness, aiming to find out the influence of various operating conditions and geometries. Taking in consideration of droplet size distribution, G. D. Chen et al. ${ }^{9}$ put forward an integrated model combined with droplet movement, droplet/wall impingement and oil film flow, in order to reveal the configuration and flow of wall oil film. W. Kurz et al. ${ }^{\mathbf{1 0}}$ believed that different flow regimes correspond to different oil film distributions inside an aero engine bearing chamber, and proposed an approach for the flow regime prediction.

In addition to the above researches on the flow characteristics, the impacts of temperature distribution and heat transfer process inside bearing chambers deserve enough attention. For this reason, M. Flouros ${ }^{\mathbf{1 1}}$ developed correlations for heat generation and outer ring temperature which consider the influence of the direction of oil ingestion relative to the direction of the axial load. On an engine demonstrator, he examined the two-phase flow heat transfer in the vent and scavenge pipe analytically and numerically. ${ }^{12}$ A further step research was carried out on the active outer ring cooling of ball bearings, by using a spiral groove engraved in the outer ring with oil as the cooling medium. ${ }^{13}$

Nowadays, aircraft engines, e.g. UAV engines, are developing towards lighter weight and more compact structure, which consequently put forward higher demands on lubrication systems, not only in terms of bearings lubrication, but also in terms of the removal of the vast amounts of heat generation. The flow field is more complicated compared to that in a common aero engine, due to high shaft rotating speed, geometric configurations and a strong interaction between air flow and lubricant oil. Therefore, it is of great significance to understand the characteristics of flow and heat transfer in UAV engine bearing chamber, so as to optimize its design and operation.

However, few literatures can be referred to regarding the above topic in UAV engines. In the present paper, based on a theoretical analysis into the main heat sources inside the chamber, a three dimensional investigation is carried out on the physical fields, namely path lines, the velocity distributions, and temperature distributions, in a turbojet engine generally applied to unmanned target drones. The impacts of operating conditions, including the rotating speed, oil supply, and air inlet velocity are determined, respectively.

\section{Modeling}

\subsection{Model set-up}

The schematic structure of a micro UAV engine is depicted in Fig. 1. An open-type system is employed, where lube oil and air are pushed into the engine periodically to make sure that the bearings and their accessories work in good condition. This system, which has simple structure as there are no mechanical components for lube circulation, is usually used in engines working at high temperature and for short time. Accordingly, the structural model built by 3D Unigraphics (UG) software is depicted in Fig. 2, including the detailed geometric parameters.

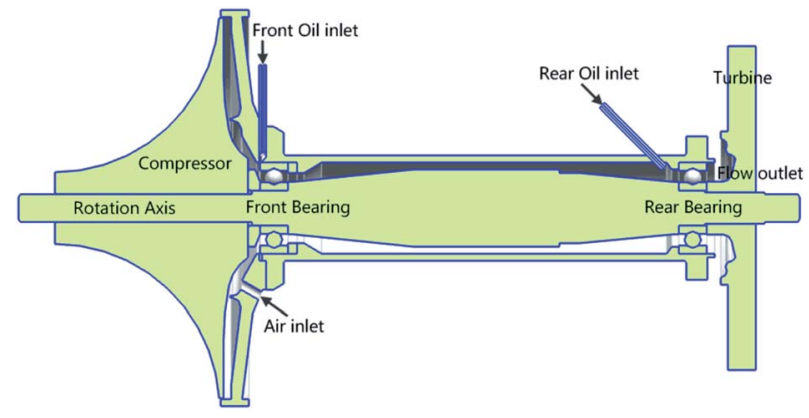

Fig. 1 Schematic of a micro UAV engine.

\subsection{Mesh generation}

The above model is then meshed by appropriate selection of structured or unstructured grids. Specifically, for the central region between the shaft and wall, and the inlet and outlet regions of lube oil, where the structures are relatively simple, structured grids are selected; while for the regions of bearings with rotors, where the structures are far more complicated, unstructured grids are more suitable.

After that, the mesh quantity is set at $1 \times 10^{6}, 1.5 \times 10^{6}, 2 \times$ $10^{6}, 2.9 \times 10^{6}$ and $3.5 \times 10^{6}$ respectively, and the average temperatures at central cross-section of the rear bearing, calculated by different mesh quantities, are presented in Fig. 3 . It can be inferred that the optimal mesh quantity is $2 \times 10^{6}$, both to save the calculation time and to ensure the simulation accuracy.

\subsection{Physical properties and boundary conditions}

Physical properties of the lube oil can be calculated by the equations below. ${ }^{\mathbf{1 4}}$

Density

$$
\rho=0.75(293-T)+779.6 \mathrm{~kg} \mathrm{~m}^{-3}
$$

Specific heat capacity

$$
C_{\mathrm{p}}=0.003762 T+0.44 \mathrm{~J} \mathrm{~kg}^{-1} \mathrm{~K}^{-1}
$$

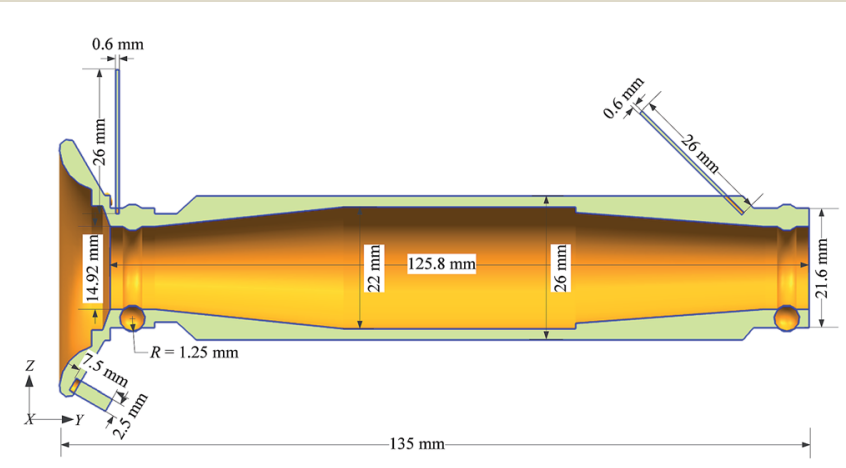

Fig. 2 Structural model of the bearing chamber by UG. 


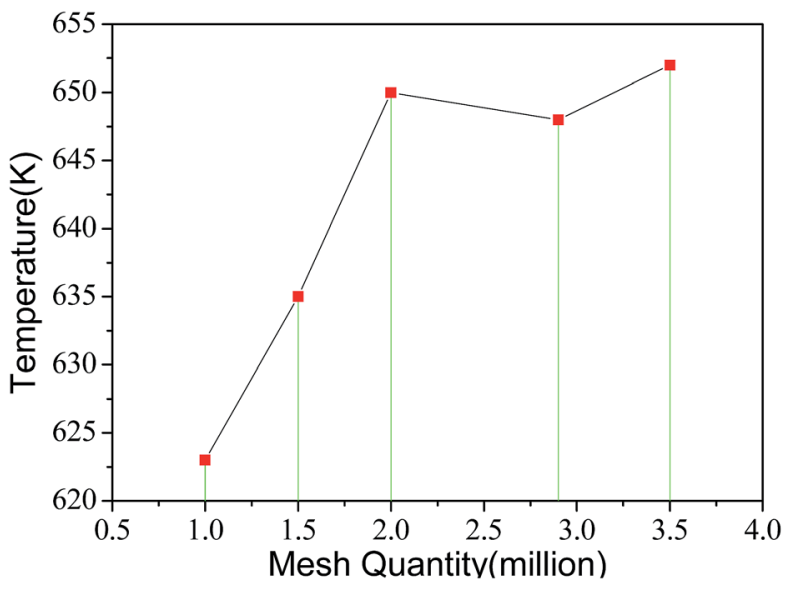

Fig. 3 The grid independence test and verification.

Heat conductivity coefficient

$$
\lambda=0.12-0.00016 T \mathrm{~W} \mathrm{~m}^{-1} \mathrm{~K}^{-1}
$$

Kinematic viscosity

$$
\lg (v+0.73)=10^{(8.9765-3.8265 \lg T)} \mathrm{mm}^{2} \mathrm{~s}^{-1}
$$

On FLUENT platform, mixture model combined with RNG $\mathrm{k}-\varepsilon$ turbulent model is employed to investigate the air-oil two phase flows in the bearing chamber of a UAV engine. Velocity boundary condition is applied at the air inlet, flow rate boundary condition is used at the oil inlets, and pressure boundary condition is applied at the outlet of bearing chamber, with total pressure of $377334 \mathrm{~Pa}$ and static pressure of 366844 Pa. Besides, SIMPLE scheme is used to resolve pressure-velocity coupling equation. Second-order upwind difference scheme is also applied to improve the computational accuracy, with the convergence accuracy $10^{-6}$ of the residual monitor.

The initial temperatures at key points are listed in Table 1. In addition, due to high-speed friction between rotors and track inside bearings, the heat production needs to be calculated, based on friction moments. ${ }^{15}$ Frictional heat production model, proposed by Palmgren of SKF (Svenska Kullagerfabriken), is employed here.

Total heat production

$$
Q=2 \pi M n 10^{-3} / 60 \mathrm{~W}
$$

Table 1 Initial temperatures at key points

\begin{tabular}{ll}
\hline Temperatures & Values (K) \\
\hline$T_{\mathrm{li}}$ & 300 \\
$T_{\mathrm{ai}}$ & 464 \\
$T_{\mathrm{ef}}$ & 464 \\
$T_{\mathrm{er}}$ & 1180
\end{tabular}

Total friction moment

$$
M=M_{0}+M_{1} \mathrm{~N} \mathrm{~mm}
$$

Viscous friction moment by lube

$$
M_{0}=10^{-7} f_{0}(v n)^{\frac{2}{3}} D_{\mathrm{m}}^{3} \mathrm{~N} \mathrm{~mm}
$$

Load moment

$$
M_{1}=f_{1} F_{1} D_{\mathrm{m}} \mathrm{N} \mathrm{mm}
$$

where $f_{0}$ is a coefficient determined by bearing type and lubrication method, $n$ is revolving speed of the bearing, $f_{1}$ is a coefficient determined by bearing structure and loads, $F_{1}$ is the calculated bearing load, and $D_{\mathrm{m}}$ is calculated by:

$$
D_{\mathrm{m}}=\frac{1}{2}\left(D_{\mathrm{i}}+D_{\mathrm{o}}\right) \mathrm{mm}
$$

$D_{\mathrm{i}}$ and $D_{\mathrm{o}}$ are inner circle diameter and outer circle diameter of the bearing, respectively.

It is assumed that each one of total 11 rotors produces the same quantity of heat in a high-speed rolling bearing, thus the heat production flux over the total heat transfer area is:

$$
q=\frac{Q}{11 \pi D_{\mathrm{a}}^{2}+\pi D_{\mathrm{i}} D_{\mathrm{a}}+\pi D_{\mathrm{o}} D_{\mathrm{a}}} \mathrm{W} \mathrm{m}{ }^{-2}
$$

where $D_{\mathrm{a}}$ is the diameter of the rotor.

\section{Results and discussion}

\subsection{Verification of the simulated results}

The typical working conditions are presented in Table 2. Numerical simulation was carried out under these conditions, and the simulation results were in good agreement with the practical measured values, shown in Table 3.

\subsection{Physical fields}

3.2.1 Path lines of the fluids. Fig. 4 depicts the path lines of the air-oil fluids along the longitudinal section $(X=0)$ in the UAV bearing chamber. Firstly, the hot air enters the bearing chamber, part of which impinges the pipe wall, forming small vortexes; the other part moves towards the right direction along the channel and mixes with the lubrication oil from the front

Table 2 Typical working conditions

\begin{tabular}{ll}
\hline Conditions & Values \\
\hline$n(\mathrm{rpm})$ & 96000 \\
$m_{\mathrm{if}}\left(\mathrm{g} \mathrm{s}^{-1}\right)$ & 4.2 \\
$m_{\mathrm{ir}}\left(\mathrm{g} \mathrm{s}^{-1}\right)$ & 8.4 \\
$T_{\mathrm{li}}(\mathrm{K})$ & 300 \\
$v_{\mathrm{ai}}\left(\mathrm{m} \mathrm{s}^{-1}\right)$ & 60 \\
$T_{\mathrm{ai}}(\mathrm{K})$ & 464 \\
$P_{\mathrm{o}}(\mathrm{Pa})$ & 366844
\end{tabular}


Table 3 Comparison of simulation results with measured values

\begin{tabular}{llll}
\hline Parameters & $\begin{array}{l}\text { Simulation } \\
\text { results }\end{array}$ & Measured values & Relative errors \\
\hline$v_{\mathrm{o}}\left(\mathrm{m} \mathrm{s}^{-1}\right)$ & 110 & 138 & $20.3 \%$ \\
$T_{\mathrm{i}}(\mathrm{K})$ & 1165 & 1180 & $1.3 \%$ \\
$T_{\mathrm{r}}(\mathrm{K})$ & 665 & 670 & $0.7 \%$
\end{tabular}

inlet, producing the air-oil two phase flow. In the following zone close to the front bearing, two or three major vortexes are observed, due to the special sudden-expanding and gradualcontracting structure at that location. Then, under the influence of high-speed shaft revolution, the fluids move forward wavelike when impinging against the walls, where several intermittent small vortexes are produced adjacent to the walls. After that, the air-oil fluids enter another sudden-expanding section which further complicates the flow by generating larger and more complex vortexes. In addition, more oil will be added through the inclined "rear oil inlet" into the channel, mixes with the air-oil fluids, and flows through the rear bearing. Finally, the air-oil two phase fluids flow out of the bearing chamber.

3.2.2 Velocity distribution. The velocity distribution along the $X=0$ longitudinal section is exhibited in Fig. 5 . It can be clearly seen that in the region ahead of the front bearing, the fluids velocity is very limited, mainly due to the obstruction of the front bearing. After the front bearing, the fluids speed up gradually on account of the differential pressure and the convergent channel. Besides, the velocity declines along the radial direction, which is understandable that the shaft rotation affects the fluids velocity greatly, and as a result, the fluids close to the shaft have a higher velocity, whereas the fluids away from the shaft have a lower velocity; especially in the zone proximity to walls, the velocity plummets due to the friction from the stationary surface. More interestingly, it is noticed that in the middle section, several intermittent lower-velocity zones close to walls and higher-velocity zones close to shaft are observed, which corresponds to the several intermittent vortexes in Fig. 4.

In the rear zone near the inclined inlet of lube oil, the velocity decreases again due to the slow inflow of oil, and it drops further when fluids flow through the rear bearing, caused by the flow resistance in the rear bearing.

3.2.3 Temperature distribution. Fig. 6 depicts the temperature distribution along the $X=0$ longitudinal section, with

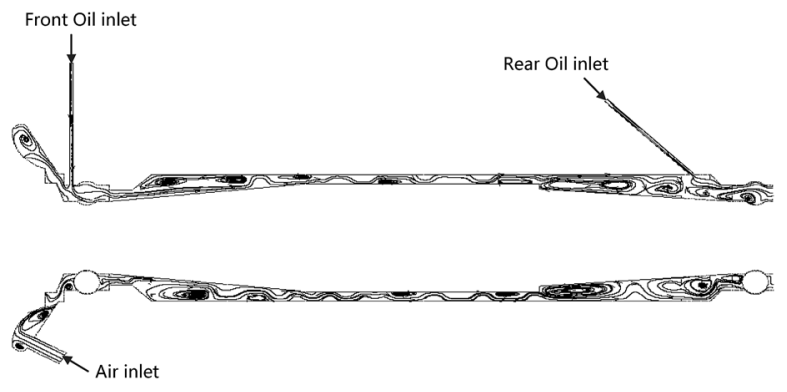

Fig. 4 Path lines of the air-oil fluids along the longitudinal section $(X=0)$.

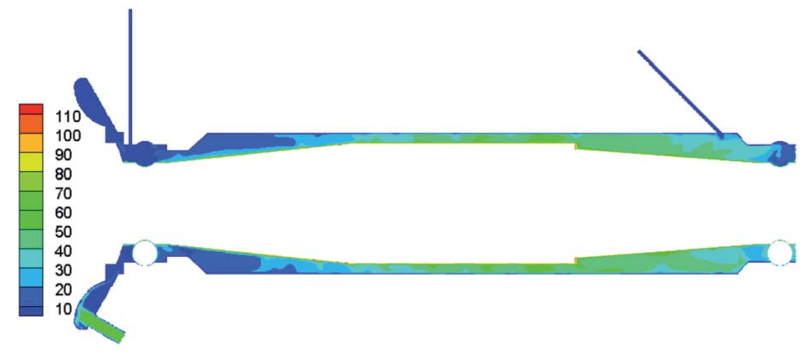

Fig. 5 Velocity $\left(\mathrm{m} \mathrm{s}^{-1}\right)$ distribution along the $X=0$ longitudinal section.

exterior surrounding temperature gradually increasing from $464 \mathrm{~K}$ at the front bearing to $1180 \mathrm{~K}$ at the rear bearing. As a result, overall the fluids temperature gradually rises along the axial direction. It is worth noting that in the middle section (M zone), several tiny higher-temperature areas are discovered, suggesting that the local heat transfer is enhanced, which can be interpreted by the impact of the intermittent small vortexes in Fig. 4. Additionally, the higher-temperature exterior surrounding, along with the friction heat of the bearing, leads to a higher temperature at the rear section (R zone).

In order to further understand the cooling effects on the two bearings, the temperature distribution along the cross section of the two bearings are separately showed in Fig. 7. Note that the highest temperature on the rear bearing is $780 \mathrm{~K}$, compared to $560 \mathrm{~K}$ on the front one, which indicates that cooling the rear bearing is more crucial for the entire bearing chamber. Moreover, Fig. 7(b) illustrates that the temperature at the inner circle is relatively low, while the highest temperature spots exist at the outer circle, since the exterior surrounding temperature is $1180 \mathrm{~K}$, which is much higher than those at other points, as presented in Table 1. By comparison, the heat source along the inner circle, which results from the friction with rotors, is of lower temperature. Therefore, in the following sections, detailed research will be conducted on how each operating condition will affect the cooling for the rear bearing.

\subsection{Influences of operating conditions}

3.3.1 Influence of shaft rotating speed. In general, as the rotating speed increases, more acting force from the shaft and rotors will increase the fluids velocity, which contributes to a better heat transfer between fluids and the bearing. On the

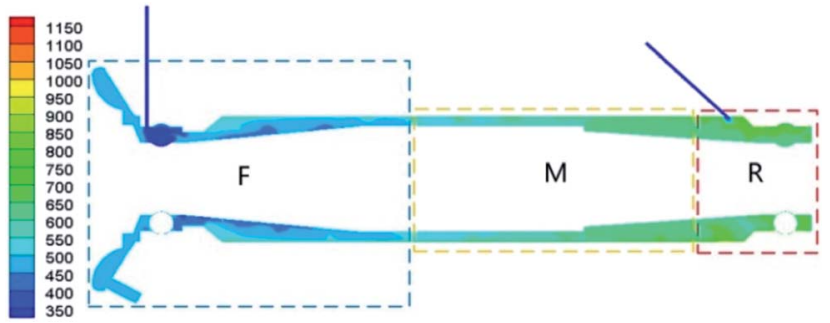

Fig. 6 Temperature $(K)$ distribution along the $X=0$ longitudinal section. 


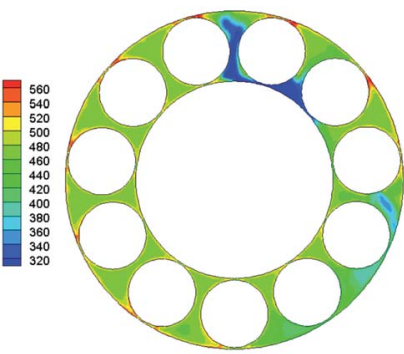

(a) Front bearing

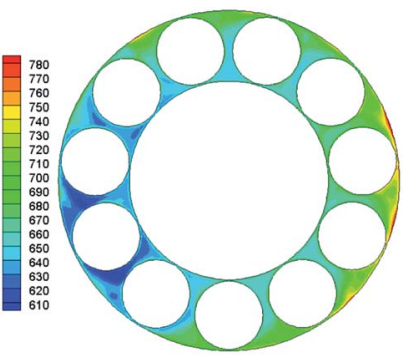

(b) Rear bearing
Fig. 7 Temperature $(K)$ distribution along the cross section of bearings.

other hand, the heat production by friction between fluids and bearing (rotors) will rise meanwhile.

At a lower revolving speed (84 $000 \mathrm{rpm}$ or $88000 \mathrm{rpm}$ ), the heat production by friction is relatively less, thus the fluids can "take away" heat from the bearing timely, in other words, the cooling effect on rotors and inner circle of the bearing is satisfactory, as it can be seen from Fig. 8(a) and (b). With the revolving speed increases to $92000 \mathrm{rpm}$ and $96000 \mathrm{rpm}$, both the heat production by friction and the heat transfer coefficient between fluids and solid walls grow consequently. Given the temperature of exterior surrounding at the rear bearing may reach up to $1180 \mathrm{~K}$ (Table 1), as a result, the fluids temperature increases, shown in Fig. 8(c) and (d).

As for the temperature distribution on the outer circle of the rear bearing, it is noticed that at a lower revolving speed $(84000 \mathrm{rpm})$, more high-temperature spots are observed, approximately 9 red spots (Fig. 8(a)), resulting from a lower heat transfer coefficient between fluids and the outer circle, compared to only 3 red high-temperature spots (Fig. 8(d)) at

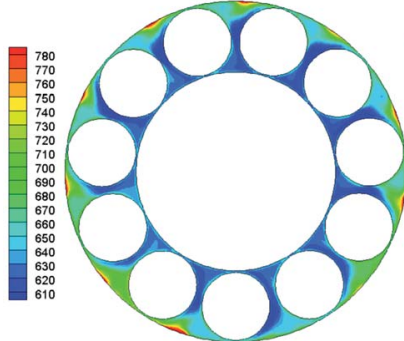

(a) $84000 \mathrm{r} \cdot \mathrm{min}^{-1}$

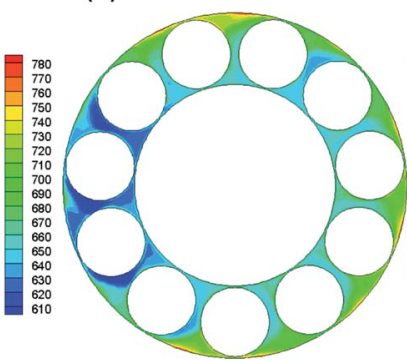

(c) $92000 \mathrm{r} \cdot \mathrm{min}^{-1}$

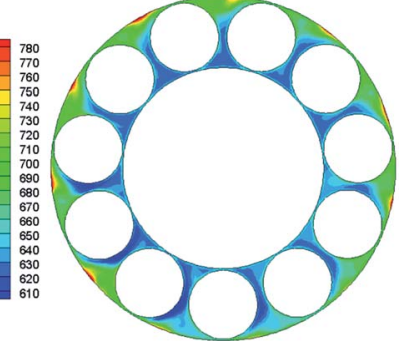

(b) $88000 \mathrm{r} \cdot \mathrm{min}^{-1}$

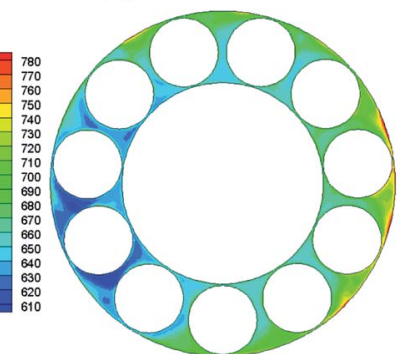

(d) $96000 \mathrm{r} \cdot \mathrm{min}^{-1}$
Fig. 8 Temperature $(K)$ distribution of the rear bearing under different revolving speed.

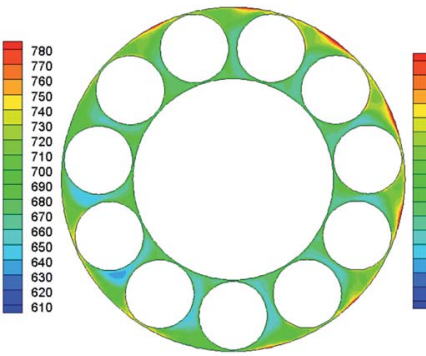

(a) $4.2 \mathrm{~g} \cdot \mathrm{s}^{-1}$

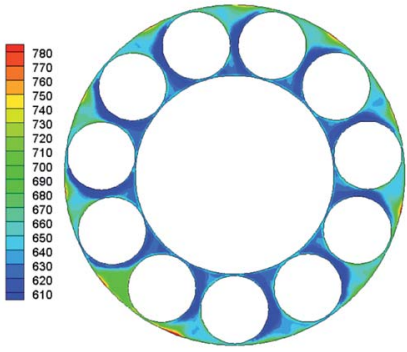

(c) $8.4 \mathrm{~g} \cdot \mathrm{s}^{-1}$

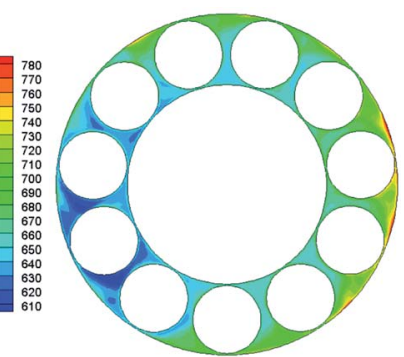

(b) $6.3 \mathrm{~g} \cdot \mathrm{s}^{-1}$

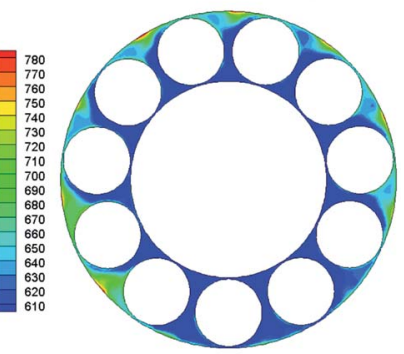

(d) $10.5 \mathrm{~g} \cdot \mathrm{s}^{-1}$
Fig. 9 Temperature $(K)$ distribution of the rear bearing under different oil supply.

a higher revolving speed (96000 rpm) when the heat transfer is much better. Moreover, with the heat transfer coefficient increasing, the temperature distribution over the cross section of the bearing is more uniform.

To summarize, an excessively high revolving speed may result in overtemperature for rotors and inner circle of the bearing. However, a lower revolving speed may cause a poor cooling effect on the outer circle of the bearing. Take the above factors into consideration, it is suggested that the optimal revolving speed be $92000 \mathrm{rpm}$.

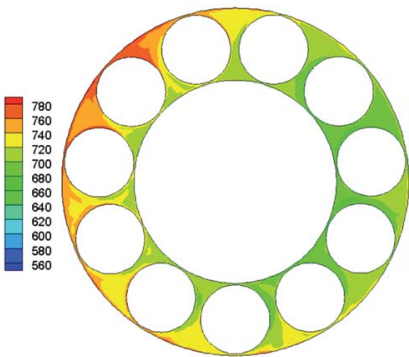

(a) $20 \mathrm{~m} \cdot \mathrm{s}^{-1}$

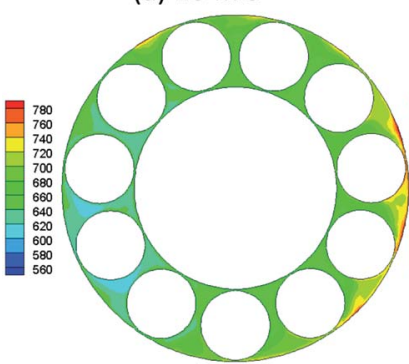

(c) $60 \mathrm{~m} \cdot \mathrm{s}^{-1}$

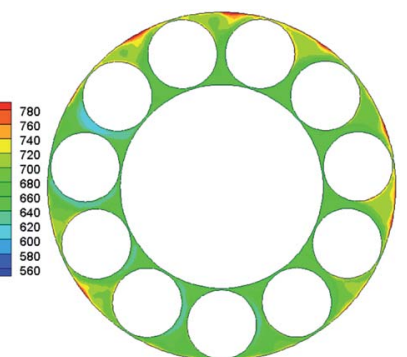

(b) $40 \mathrm{~m} \cdot \mathrm{s}^{-1}$

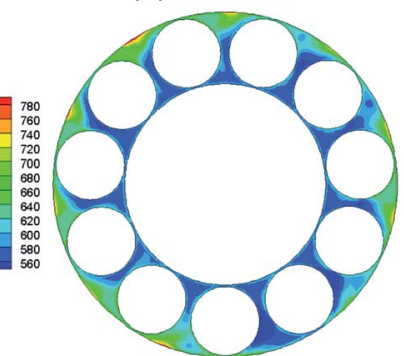

(d) $80 \mathrm{~m} \cdot \mathrm{s}^{-1}$
Fig. 10 Temperature $(K)$ distribution of the rear bearing under different air inlet velocity. 
Table 4 Key temperatures of the rear chamber under different air inlet velocity

\begin{tabular}{lllll}
\hline$v_{\text {ai }}\left(\mathrm{m} \mathrm{s}^{-1}\right)$ & $T_{\mathrm{m}}(\mathrm{K})$ & $T_{\text {ic }}(\mathrm{K})$ & $T_{\text {oc }}(\mathrm{K})$ & $T_{\mathrm{r}}(\mathrm{K})$ \\
\hline 20 & 717 & 708 & 743 & 718 \\
40 & 672 & 671 & 730 & 683 \\
60 & 665 & 667 & 715 & 678 \\
80 & 600 & 595 & 698 & 608 \\
100 & 587 & 585 & 680 & 600 \\
120 & 580 & 578 & 670 & 589
\end{tabular}

3.3.2 Influence of oil supply. The rear lubrication oil supply affects the temperature distribution of the rear bearing significantly, as clearly depicted in Fig. 9. In other words, the lube oil supply plays an important role in cooling bearings. In general, more lube supply will contribute to better cooling effect on the bearing. Nevertheless, too much lube supply will consume more power and increase load on the engine, which consequently reduce the engine efficiency. In the present work, an optimal oil supply is suggested around $8.4 \mathrm{~g} \mathrm{~s}^{-1}$.

3.3.3 Influence of air inlet velocity. Raising the air inlet velocity is also beneficial to the bearing cooling, as shown in Fig. 10. At a low air inlet velocity of $20 \mathrm{~m} \mathrm{~s}^{-1}$, the noticeable red high-temperature areas are observed in Fig. 10(a), which suggests that the bearing is working under an undesirable condition with poor cooling effect. With the air inlet velocity increasing from $20 \mathrm{~m} \mathrm{~s}^{-1}$ to $80 \mathrm{~m} \mathrm{~s}^{-1}$, red spots diminishes dramatically, because the increase of air inlet velocity speeds up the air-oil two phase flow, and as a result, the heat transfer coefficient between fluids and the bearing increases, which leads to a better cooling condition. Nevertheless, it should be pointed out that a further increase of the air inlet velocity to over $80 \mathrm{~m} \mathrm{~s}^{-1}$ does not necessarily mean a steady enhancement of cooling effect, as presented in Table 4, instead, too much air supply may degrade the fluids lubrication. Therefore, taking the above two factors into account, the optimal air inlet velocity for this UAV engine is around $80 \mathrm{~m} \mathrm{~s}^{-1}$.

\section{Conclusions}

High shaft rotating speed of micro UAV engines often causes a poor cooling effect. It is necessary to investigate the characteristics of flow and heat transfer in the bearing chamber and to understand the impacting factors. In the present paper, a thermal-fluid coupling numerical study is carried out on FLUENT Platform for a certain type of micro UAV engine. Some key physical fields, such as path lines, velocity and temperature distributions were obtained and discussed. Based on that, the impacts of revolving speed, oil supply, and air inlet velocity were respectively determined, with the conclusion as follows:

(1) Path lines of the fluids along the bearing chamber feature vortexes of different sizes at different locations, due to the effects of local channel geometry, interaction between air and oil, collisions between fluids and walls, and oil injection.

(2) The velocity distribution is mainly influenced by the obstruction of bearings, channel geometry, the radial distance to the revolving shaft, and the friction from the stationary wall, etc. Similarly, the temperatures in the bearing chamber are affected by the exterior surrounding temperature, the frictional heat production of bearings, and the radial location in bearings.

(3) Both the velocity distribution and the temperature distribution are closely associated with the path lines. More specifically, the vortexes of fluids in the figure of path lines have immediate impacts on the local velocity and temperature.

(4) Shaft rotating speed determines the frictional heat production of rotors, and the cooling effect on the outer circle of the bearing as well. In general, more lubrication oil supply contributes to better cooling effect on the bearing; however, it will meanwhile consume more power and reduce the engine efficiency. Additionally, raising the air velocity will, on the one hand, be beneficial to the bearings' cooling; on the other hand, degrade the fluids lubrication capability.

(5) Taking into consideration of the cooling effect, lubrication effect and engine efficiency, the suggested values for rotating speed, lubrication oil supply and air velocity are around $92000 \mathrm{rpm}, 8.4 \mathrm{~g} \mathrm{~s}^{-1}$ and $80 \mathrm{~m} \mathrm{~s}^{-1}$ respectively, with respect to the micro UAV engine in the present paper.

\section{Conflicts of interest}

There are no conflicts to declare.

\section{Nomenclature}

$C_{\mathrm{p}} \quad$ Specific heat capacity of lube oil

$D_{\mathrm{a}} \quad$ Diameter of the rotor

$D_{\mathrm{i}} \quad$ Inner circle diameter of the bearing

$D_{\mathrm{m}} \quad$ Circle diameter of bearing pitch

$D_{\text {o }} \quad$ Outer circle diameter of the bearing

$f_{0} \quad$ Coefficient determined by bearing type and lubrication

$f_{1} \quad$ Coefficient determined by bearing structure and loads

$F_{1} \quad$ Bearing load

$m_{\text {if }} \quad$ Inlet mass flow rate of lube at the front bearing

$m_{\text {ir }} \quad$ Inlet mass flow rate of lube at the rear bearing

$M \quad$ Total friction moment

$M_{0} \quad$ Viscous friction moment by lube

$M_{1} \quad$ Load moment

$N \quad$ Revolving speed of the bearing

$P_{\mathrm{o}} \quad$ Outlet static pressure of fluids

$Q \quad$ Heat production flux

$Q \quad$ Total heat production

$T \quad$ Temperature of lube oil

$T_{\text {ai }} \quad$ Inlet air temperature

$T_{\text {ef }} \quad$ Temperature of exterior surrounding at the front

$T_{\mathrm{er}} \quad$ Temperature of exterior surrounding at the rear

$T_{\text {ic }} \quad$ Average temperature on the inner circle

$T_{\text {li }} \quad$ Inlet lube temperature

$T_{\mathrm{m}} \quad$ Average temperature of fluids on the cross section

$T_{\mathrm{o}} \quad$ Outlet temperature the fluids

$T_{\mathrm{oc}} \quad$ Average temperature on the outer circle

$T_{\mathrm{r}} \quad$ Average temperature on rotors

$v_{\text {ai }} \quad$ Inlet air velocity

$v_{\mathrm{o}} \quad$ Outlet velocity of the fluids

$X \quad$ Axis 


\section{Greek symbols}

$\begin{array}{ll}\rho & \text { Density of lube oil } \\ \Lambda & \text { Heat conductivity coefficient of lube oil } \\ \nu & \text { Kinematic viscosity of lube oil }\end{array}$

\section{Acknowledgements}

This work is supported by "the Fundamental Research Funds for the Central Universities" (No. NJ20160041); and "National Natural Science Foundation of China” (No. 51506087).

\section{References}

1 C. W. Lee, P. C. Palma, K. Simmons and S. J. Pickering, J. Eng. Gas Turbines Power, 2005, 127, 697-703.

2 J. Aidarinis, D. Missirlis, K. Yakinthos and A. Goulas, J. Eng. Gas Turbines Power, 2011, 133, 082504.

3 M. Farrall, S. Hibberd, K. Simmons and D. Giddings, Proc. Inst. Mech. Eng., Part G, 2006, 220, 197-202.
4 B. Chen, G. Chen, H. Sun and Y. Zhang, Proc. Inst. Mech. Eng., Part G, 2012, 228, 206-218.

5 H. C. Sun, G. D. Chen, L. N. Wang and F. Wang, Int. J. Aerosp. Eng., 2015, 769862.

6 H. C. Sun, G. D. Chen, Y. H. Zhang and L. N. Wang, Proc. Inst. Mech. Eng., Part G, 2016, 230, 2596-2614.

7 M. Farrall, S. Hibberd and K. Simmons, J. Eng. Gas Turbines Power, 2008, 130, 012501.

8 P. Gorse, S. Busam and K. Dullenkopf, J. Eng. Gas Turbines Power, 2006, 128, 103-110.

9 G. D. Chen, H. C. Sun and J. Wang, Chin. J. Aeronaut., 2011, 24, 355-362.

10 W. Kurz and H. J. Bauer, Proceedings of the Asme Turbo Expo: Turbine Technical Conference and Exposition, Dusseldorf, 2014, pp. 1-8.

11 M. Flouros, Aerosp. Sci. Technol., 2006, 10, 611-617.

12 M. Flouros, J. Turbomach., 2010, 132, 011008.

13 M. Flouros, M. Hirschmann, F. Cottier, P. Gloeckner and K. Dullenkopf, J. Eng. Gas Turbines Power, 2013, 135, 081902.

14 S. H. Tong, Master, Nanjing University of Aeronautics and Astronautics, 2013.

15 Y. C. Zhou, Master, Lanzhou University of Technology, 2013. 